\title{
RNF180 Suppresses Methylation of ADAMTS9 DNA Promotor by Ubiquitinating DNMT3A Inhibiting Metastasis in Gastric Cancer
}

\section{Weilin Sun}

Tianjin tumor hospital

Jingyu Deng ( $\sim$ dengery@126.com )

Tianjin Tumor Hospital

\section{Gang Ma}

Tianjin Tumor Hospital

\section{Li Zhang}

Tianjin Tumor Hospital

\section{Pengliang Wang}

Tianjin Tumor Hospital

Huifang Liu

Tianjin tumor hospital

Nannan Zhang

Tianjin Tumor Hospital

Zizhen Wu

Tianjin Tumor Hospital

\section{Yinping Dong}

Tianjin tumor hospital

\section{Fenglin Cai}

Tianjin Tumor Hospital

\section{Liqiao Chen}

Tianjin Tumor Hospital

\section{Zhenzhen Zhao}

Tianjin tumor hospital

Han Liang

Tianjin tumor hospital

\section{Research}

Keywords: ADAMTS9, ring finger protein 180, gastric cancer, metastasis 
DOI: https://doi.org/10.21203/rs.3.rs-30779/v1

License: (c) (i) This work is licensed under a Creative Commons Attribution 4.0 International License. Read Full License 


\section{Abstract}

BACKGROUND: A disintegrin-like and metalloprotease with thrombospondin type 1 motif 9 (ADAMTS9) is hypermethylated and inhibits the proliferation of various cancers. In this study, we demonstrated that the expression of ADAMTS9 was associated with the lymphatic metastasis of gastric cancer (GC) and elucidated the down- and upstream molecular pathways in GC progression.

METHODS: The study explored the expression level, biological function, clinical application, and involved molecular mechanism of ADAMTS9 in GC.

RESULTS: In 135 GC tissue samples, ADAMTS9 expression level was significantly correlated with the pN stage, the number of metastatic lymph nodes (LNs), and the overall survival of patients with GC. The in vitro and in vivo experiments showed that ADAMTS9 attenuated the viability and motile capacity of GC cells. Mechanistic investigations revealed that ADAMTS9 significantly inhibited the transcription of $C-C$ motif chemokine ligand 5 (CCL5)/C-X-C motif chemokine ligand 11 (CXCL11). This effect impaired the migration and invasion in GC cells. This study revealed that the hypermethylation level in the promotor of ADAMTS9 gene was mainly mediated by DNA-methyltransferase(DNMT) 3A, which reduces ADAMTS9 expression in GC. Ring finger protein (RNF) 180 could promote DNMT3Aubiquitination and degradation, thereby restoring the ADAMTS9 expression in GC cells.

CONCLUSIONS: ADAMTS9 expression is restored by the RNF180 via suppressing the promotor methylation of the ADAMTS9 gene. ADAMTS9 inhibits metastasis and improves the prognosis of patients with GC via CCL5/CXCL11-dependent pathway. Thus, ADAMTS9 should be considered as a predictor of LN metastasis and a therapeutic target in GC.

\section{Background》}

Gastric cancer (GC) is currently the 5th most common malignant tumor and the 3rd leading cause of cancer-related death ${ }^{1}$. GC shows a strong tendency to spread locally and metastasize via the lymph nodes $(\mathrm{LN})$ because of the abundant blood and lymphatic vessels in the gastric area. Although surgery has significantly improved the prognosis of patients, the wide extent of LN metastasis always limits the management and treatment of patients with GC, thereby leading to a poor 5 -year survival rate of $20 \%-30 \%$ in patients with advanced $\mathrm{GC}^{2}$. Thus, tracking and identifying a novel effective biomarker that contributes to metastasis remains imperative for GC.

A disintegrin-like and metalloproteinase with thrombospondin motifs (ADAMTS) is a family of secreted, multi-domain matrix-associated zinc metalloendopeptidases with 19 members ${ }^{3}$. ADAMTS family proteins are originally identified as the cleave-type substrates in the extracellular matrix. These proteins participate in numerous physiological processes and diseases, such as cardiovascular disease ${ }^{4}$, arthritis ${ }^{5}$, cancer ${ }^{6}$, and angiogenesis ${ }^{7}$. ADAMTS 9 is a member of the ADAMTS family that acts as a tumor suppressor in several types of human cancers, such as esophagea ${ }^{8}$, nasopharyngeal ${ }^{9}$, breast $^{10}$ and colorectal 
cancers $^{11}$, and $\mathrm{GC}^{12}$. However, the correlation between ADAMTS9 and LN metastases in GC has been rarely reported, and the downstream molecular pathway remains unclear.

The promotor methylation of tumor-suppressor genes or tumor-related genes is usually involved in the multistep progression of tumor carcinogenesis and the development of cancer ${ }^{13}$. The hypermethylation of the promotor of ADAMTS9 gene is the most important cause that decreases or silences the expression of ADAMTS $9^{12}$. The mechanism of modulating the promotor methylation of ADAMTS9 gene remains unclear. The novel E3 ubiquitin ligase involved in protein ubiquitination process, ring finger protein (RNF) 180 is a tumor suppressor in $\mathrm{GC}^{14}$. Ubiquitination plays an essential role in protein post-translation modification and is involved in various processes of tumorigenesis and tumor development ${ }^{15}$. Identifying the substrate of E3 ubiquitin ligase and improving the understanding of the ubiquitination process benefit clinicians in tumor treatment. Through the correlation analysis from the GEPIA database (http://gepia.cancer-pku.cn/), we found that the mRNA expression level of RNF180 might have a positive correlation with ADAMTS9. In addition, the immunohistochemistry (IHC) staining of tissue microarrays (TMAs) with $135 \mathrm{GC}$ tissue samples also showed a significant positive correlation between RNF180 and ADAMTS9 expression. Thus, we hypothesized that RNF180 may suppress the methylation of ADAMTS9 DNA promotor to restore the ADAMTS9 expression in GC. In this study, we initially explored the interactional substrate of RNF180 and revealed the potential molecular mechanism of mediating ADAMTS9 expression in GC.

In present study, we demonstrated that the low expression of ADAMTS9 was closely associated with advanced $\mathrm{pN}$ stage and poor survival outcome in GC. ADAMTS9 attenuated the viability and motile capacity of GC cells. We uncovered that ADAMTS9 waspartly located in the cell nucleus region and inhibited the transcription of chemokine ligand 5 (CCL5) and C-X-C motif chemokine ligand 11 (CXCL11). This effect impaired the migration and invasion behavior of GC. ADAMTS9 expression was mainly regulated by the promotor methylation level via modifying the expressions of DNA-methyltransferase (DNMT)3A. RNF180 promotes DNMT3Aubiquitination and degradation, thereby restoring the expression of ADAMTS9 in GC. Thus, our study identified ADAMTS9 as the potential target for the prediction of LN metastasis and prognosis and elucidated the potential up- and downstream molecular pathways in GC.

\section{Methods:}

\section{Patients and tissue samples}

After curative gastrectomy, 135 pairs of GC tissues and matched adjacent non-tumor tissues were retrieved from the Department of Gastroenterology, Tianjin Medical University Cancer Institute and Hospital (Tianjin, China) between August 2004 and December 2007. These matched tissues were then sent to Shanghai Outdo Biotech Company (Shanghai China) for TMAs (Cat No. T14-501 TMA1-3). Tissue total RNA was extracted from 16 pairs of randomly selected GC tissue and matched adjacent non-tumor tissues after gastrectomy between July 2018 and December 2018. All tumor and adjacent non-tumor tissue samples were histologically verified. The patients in this study were not subjected to radiation, chemical, or 
biological treatment before potential curative gastrectomy. Adjuvant chemotherapy or radiotherapy was not routinely administered to the patients. The clinicopathological characteristics of the two cohorts are summarized in Table 1 and Supplementary Table S1, respectively. Patient consent was obtained for the use of the tissue samples. The study protocol and permission for the use of the clinical data were given by the Institutional Research Ethics Committee of Tianjin Medical University Cancer Institute and Hospital (Tianjin, China). 
Table 1

Clinicopathologic Features of ADAMTS9 Expression in Gastric Cancer Tissues

\begin{tabular}{|c|c|c|c|c|}
\hline \multirow[t]{2}{*}{ Characteristics } & \multicolumn{2}{|l|}{ ADAMTS9 } & \multirow[t]{2}{*}{$c^{2}$ value } & \multirow[t]{2}{*}{$P$ value } \\
\hline & High expression & Low expression & & \\
\hline \multicolumn{5}{|l|}{ Gender } \\
\hline Male & 45 & 45 & 0.018 & 0.894 \\
\hline Female & 19 & 20 & & \\
\hline \multicolumn{5}{|l|}{ Age } \\
\hline$<60$ & 33 & 37 & 0.373 & 0.541 \\
\hline$\geq 60$ & 31 & 28 & & \\
\hline \multicolumn{5}{|l|}{ Tumor location } \\
\hline Upper third & 13 & 10 & 1.830 & 0.608 \\
\hline Middle third & 8 & 5 & & \\
\hline Lower third & 29 & 36 & & \\
\hline More than $2 / 3$ stomach & 14 & 14 & & \\
\hline \multicolumn{5}{|l|}{ Tumor size } \\
\hline$<5 \mathrm{~cm}$ & 25 & 27 & 0.082 & 0.774 \\
\hline$\geq 5 \mathrm{~cm}$ & 39 & 38 & & \\
\hline \multicolumn{5}{|l|}{ pT stage } \\
\hline pT2 & 5 & 4 & 0.326 & 0.850 \\
\hline рT3 & 4 & 3 & & \\
\hline pT4 & 55 & 58 & & \\
\hline \multicolumn{5}{|l|}{ pN stge } \\
\hline pNO & 18 & 10 & 10.043 & $0.018^{*}$ \\
\hline pN1 & 9 & 4 & & \\
\hline $\mathrm{pN} 2$ & 15 & 11 & & \\
\hline pN3 & 22 & 40 & & \\
\hline pTNM stage & & & & \\
\hline
\end{tabular}

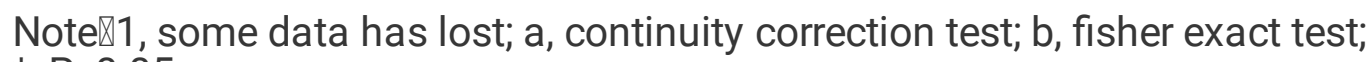
$*, P<0.05$; 


\begin{tabular}{|c|c|c|c|c|}
\hline 1 & 3 & 1 & 2.480 & $0.285^{\mathrm{b}}$ \\
\hline II & 18 & 13 & & \\
\hline III & 43 & 51 & & \\
\hline \multicolumn{5}{|l|}{ Lauren type } \\
\hline Intestinal & 13 & 12 & 3.066 & 0.216 \\
\hline Diffuse & 46 & 52 & & \\
\hline Mixed & 5 & 1 & & \\
\hline \multicolumn{5}{|l|}{ Borrman type ${ }^{1}$} \\
\hline प & 0 & 1 & 3.271 & 0.352 \\
\hline 口 & 9 & 14 & & \\
\hline 0 & 33 & 25 & & \\
\hline 0 & 5 & 6 & & \\
\hline $\begin{array}{l}\text { Note } \otimes 1 \text {, some d } \\
\star, P<0.05 ;\end{array}$ & ntin & & est; & \\
\hline
\end{tabular}

\section{Follow-up}

After curative surgery, follow-up was performed for all patients every 3-6 months for 2 years, after which follow-up was performed every year, until death, or until deadline. The median follow-up for the entire cohort was 34.0 months (range: 2-75). The follow-up of all patients who were included in this study was completed in September 2012. B ultrasonography, CT scans, chest X-ray, and endoscopy were obtained with every visit.

\section{IHC}

IHC was performed on the TMAs of resected specimens. The $1.5 \mathrm{~mm}$-diameter tissue cores from randomly selected GC tissues and matched adjacent non-tumor tissues were used for TMA preparation. The antibodies are as shown in Supplementary Table S2. The staining intensities ( 0 , negative; $1+$, weak; $2+$, moderate; and $3+$, strong) were recorded. The percentage of immunostaining and the cytoplasmic expression were assessed by $\mathrm{H}$-score system. The formula for the $\mathrm{H}$-score is: histoscore $=\Sigma(\mathrm{I} \times \mathrm{Pi})$, where $\mathrm{I}$ $=$ intensity of staining and $\mathrm{Pi}=$ percentage of stained tumor cells, producing a cytoplasmic score ranging from 0 to 300 .

\section{Cycloheximide (CHX) pulse-chase assay and MG132 assay}


To detect the half-life of DNMT3A, we treated the stable transfected AGS and BGC-823 cells with CHX (100 $\mathrm{mg} / \mathrm{mL}$ ) for the indicated times. MG132 assay was performed to determine the degradation via proteasome. AGS and BGC-823 cells were incubated with proteasome inhibitor MG132 (10 $\mu \mathrm{M})$ for $12 \mathrm{~h}$. Western blot analysis was then performed to detect DNMT3A expressions.

\section{Co-immunoprecipitation assay}

HEK293T cells were seeded into 6-well plates and transiently transfected with empty vector, pCMVRNF180-myc plasmid, or pCMV-DNMT3A-flag plasmid. After transfection for $36 \mathrm{~h}$, cells were treated with $10 \mathrm{mM}$ MG132 for $12 \mathrm{~h}$. Total proteins were then extracted using Nonidet P-40 lysis buffer supplemented with PSMF (\#8553, cst). Immunoprecipitation Kit-DYKDDDDK (Flag®) Tag Immunomagnetic Beads (TB101274, Sino Biological, North Wales, PA, USA) and Immunoprecipitation Kit -MYC Tag Immunomagnetic Beads (TB100029, Sino Biological, North Wales, PA, USA) were adopted for collecting the immunoprecipitated proteins. The immunoprecipitated proteins were measured by the Western blot analysis.

\section{Ubiquitination assay}

To evaluate the ubiquitination of DNMT3A, we transiently transfected empty vector, pCMV-DNMT3A-flag plasmid, pCMV-RNF180-myc plasmid or pCMV-HA-UB plasmid the HEK293T cells. After transfection for 36 h, HEK293T cells were incubated with MG132 $(10 \mu \mathrm{M})$ for $12 \mathrm{~h}$. The correlated proteins were extracted and analyzed by the co-immunoprecipitation assay.

\section{Genome-wide mRNA sequencing analysis}

BGC-823 cells were stably transfected with pCNDA3.1-ADAMTS9 vector or pCDNA3.1 empty vector. Total RNA was then extracted using the mirVana miRNA Isolation Kit (Ambion, Foster City, CA, USA) according to the manufacturer's protocol. RNA integrity was evaluated using the Agilent 2100 Bioanalyzer (Agilent Technologies, Santa Clara, CA, USA). The samples with RNA integrity number $\geq 7$ were subjected to subsequent analysis. The libraries were constructed using TruSeq Stranded mRNA LTSample Prep Kit (Illumina, San Diego, CA, USA) according to the manufacturer's instructions. P value $<0.05$ and foldchange $>1.50$ or $<0.67$ were set as the threshold for significantly different expression. The hierarchical cluster analysis of differentially expressed genes (DEGs) was performed to explore gene expression patterns. The GO enrichment and KEGG pathway enrichment analysis of DEGs were performed using the R software ${ }^{16}$. Transcriptome sequencing and analysis were conducted by OE Biotech Co., Ltd. (Shanghai, China).

\section{MassARRY analysis of the methylation level of ADAMTS9}


Total DNA were extracted using the DNA sample Kit (TIANGEN Biotech, Beijing, China) following the manufacturer's protocol. The quantitative DNA methylation analysis of ADAMTS9 was then performed using the MassARRAY platform (Agena; BioMiao Biological Technology, Beijing, China). The MassARRAY Compact MALDI-TOF (Agena; BioMiao Biological Technology, Beijing, China) was used to collect the mass spectra, and the EpiTYPER software (Agena; San Diego, CA) was used to calculate the methylation ratio.

Other methods and statistical analysis are available in the Supplementary dataset.

\section{Results}

\section{ADAMTS9 was associated with LN metastases and survival outcome in patients with GC}

We examined the mRNA levels of ADAMTS9 in the 16 pairs of tumor and adjacent non-tumor tissues. A total of 9/16 of tumor tissues showed lower mRNA expression level compared with that of adjacent nontumor tissues (Figure 1A), which were classified into low expression group. The correlation between the clinicopathological characteristics and mRNA expression of ADAMTS9 was analyzed. ADAMTS9 mRNA expression was significantly associated with $p N$ stage $(P=0.044)$ and lauren type $(P=0.019)$

(Supplementary Table S1). Patients with low ADAMTS9 mRNA expression were at high risk with increased metastatic LNs (12.89 \pm 3.49 vs $2.571 \pm 1.020, \mathrm{P}=0.024$, Figure 1B).

We further examined the protein expression of ADAMTS9 in GC tissues. With the IHC staining, ADAMTS9 protein expression was found in the cytoplasm and nucleus of the gastric tissue cells (Figures $1 \mathrm{C}$ and $3 \mathrm{~A}$ ). $\mathrm{H}$-score was used to evaluate the ADAMTS9 protein expression. A total of 111 pairs of GC tissues and adjacent non-tumor tissues and additional $18 \mathrm{GC}$ tissues were completely saved and well stained. Compared with that of adjacent non-tumor tissue, the H-Score of GC tissues was significantly decreased ( $P=0.008$, Figure 1D). Exactly $129 \mathrm{GC}$ tissues were then divided into the low and high expression groups according to the median of $\mathrm{H}$-score as the cut-off value. The clinicopathological characteristics are shown inTable 1. Univariate analysis suggested that patients with low ADAMTS9 expression was associated with advanced $p N$ stage $(P=0.018)$. We further examined the number of $L N s$ involvement between the two groups, and the number of metastatic $L N s$ of high expression group was significantly decreased (11.38 \pm 11.64 vs $6.73 \pm 7.63, \mathrm{P}=0.007$; Figure $1 \mathrm{E})$. ADAMTS9 expression was closely correlated to the LN metastasis.

To evaluate the prognostic value of ADAMTS9 expression in GC, we employed the Kaplan-Meier analysis.Figure $1 \mathrm{~F}$ shows that patients with GC with low ADAMTS9 expression had a significantly poorer 5 -year survival rate than those with high expression $(P=0.007)$. To remove the various interference factors, we also acquired the multivariate Cox regression analysis. In multivariate Cox regression analysis, ADAMTS9 expression level (HR 1.602, 95\% Cl 1.069-2.399, $\mathrm{P}=0.022)$ was an independent predictor of the prognosis of patients with $\mathrm{GC}$ with the lowest Akaike information criterion (AIC, AIC=71.555) and Bayesian information criterion (BIC, $\mathrm{BIC}=88.714$, Table 2). We demonstrated the crucial effect on the survival 
outcomes through stratification by pN stage. In the subgroup of patients with pN3 stage, ADAMTS9 expression was significantly associated with survival outcomes ( $P=0.011$,Figure 1F). 
Table 2

Univariate and multivariate Cox proportional Hazard models for overall survival of gastric cancer patients

Predictor
Univariate Analysis

$\mathrm{HR}(95 \% \mathrm{Cl})$
Multivariate Analysis

$\operatorname{HR}(95 \% \mathrm{Cl}) \quad \mathrm{P}$

\section{Gender}

Female vs male $\begin{array}{ll}1.372(0.905- & 0.136 \\ 2.079)\end{array}$

Age

\begin{tabular}{|c|c|c|c|}
\hline$\geq 60$ vs $<60$ & $\begin{array}{l}1.514(1.012- \\
2.245)\end{array}$ & $0.039^{*}$ & $\begin{array}{l}1.528(1.005- \\
2.323)\end{array}$ \\
\hline
\end{tabular}

\section{Tumor size}

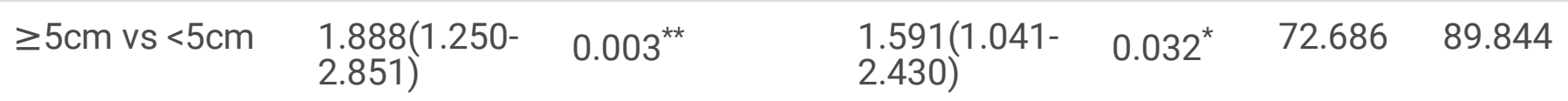

\section{Tmuor location}

Middle $1 / 3$ vs $\quad 1.232(0.588-\quad 0.580$

up $1 / 3 \quad 2.584$ )

Low 1.3 vs up $\quad 0.948(0.545-\quad 0.851$

$1 / 3 \quad 1.649)$

$>2 / 3$ stomach $1.235(0.661-0.508$

vs up1/3 2.307)

\section{Lauren type}

\begin{tabular}{lll}
$\begin{array}{l}\text { Diffuse vs } \\
\text { intestinal }\end{array}$ & $\begin{array}{l}1.144(0.677- \\
1.934)\end{array}$ & 0.615 \\
\hline Mixed vs & $\begin{array}{l}1.621(0.637- \\
\text { intestinal }\end{array}$ & 0.311 \\
\hline
\end{tabular}

\section{Brrmann type}

$\begin{array}{lll}\text { II vs I } & \begin{array}{l}0.552(0.073- \\ 4.173)\end{array} & 0.565 \\ & & \\ \text { III vs I } & \begin{array}{l}0.385(0.052- \\ 2.847)\end{array} & 0.350 \\ & \begin{array}{ll}0.512(0.064- & 0.529 \\ \text { IV vs I } & 4.113)\end{array} & \\ & \end{array}$

\section{pT stage}

Note:AIC, Akaike information criterion; BIC, Bayesian information criterion;

*,P<0.05; **, $P<0.01 ; * \star \star, P<0.001$. 


\begin{tabular}{|c|c|c|c|c|c|c|}
\hline pT3 vs pT2 & $\begin{array}{l}0.547(0.164- \\
1.821)\end{array}$ & 0.326 & & & & \\
\hline pT4 vs pT2 & $\begin{array}{l}0.788(0.381- \\
1.628)\end{array}$ & 0.519 & & & & \\
\hline \multicolumn{7}{|l|}{ pN stage } \\
\hline pN1c vs pN0 & $\begin{array}{l}0.958(0.397- \\
2.315)\end{array}$ & 0.925 & $\begin{array}{l}0.784(0.319- \\
1.929)\end{array}$ & 0.597 & 81.240 & 92.680 \\
\hline pN2 vs pNO & $\begin{array}{l}1.745(0.911- \\
3.341)\end{array}$ & 0.093 & $\begin{array}{l}1.480(0.758- \\
2.889)\end{array}$ & 0.251 & & \\
\hline pN3 vs pNO & $\begin{array}{l}2.828(1.638- \\
4.883)\end{array}$ & $<0.001^{\star \star \star}$ & $\begin{array}{l}2.231(1.273- \\
3.912)\end{array}$ & $0.005^{\star *}$ & & \\
\hline \multicolumn{7}{|c|}{ Expression of ADAMTS9 } \\
\hline Low vs high & $\begin{array}{l}1.732(1.166- \\
2.573)\end{array}$ & $0.009^{\star \star}$ & $\begin{array}{l}1.602(1.069- \\
2.399)\end{array}$ & $0.022^{*}$ & 71.555 & 88.714 \\
\hline
\end{tabular}

Note:AIC, Akaike information criterion; BIC, Bayesian information criterion;



\section{Low-expression of ADAMTS9 in GC cell lines}

The ADAMTS 9 mRNA expression in 10 types of human GC cell lines and normal GES-1 was determined. Excluding SNU-1 and HGC-27, the mRNA expression of ADAMTS9 in 8/10 GC cell lines was lower than that in GES-1 (Figure 2A). To confirm whether the protein expression follows its mRNA expression, we tested seven GC cell lines and GES-1. Western blot analysis results showed that ADAMTS9 protein was silenced or down-regulated in 6/7 GC cell lines (Figure 2B), which was consistent with their mRNA expression. The low ADAMTS9 expression in most GC cell lines might be a tumor suppressor.

\section{ADAMTS9 suppressed GC cell proliferation and viability}

To explore the biological function of ADAMTS9 in GC cells, we overexpressed ADAMTS9 in AGS, BGC-823, and SGC-7901 cells through the pCNDA3.1-ADAMTS9 plasmid. The mRNA and protein expression of ADAMTS9 were proven by RT-PCR and Western blot analysis (Figure $\mathbf{2 c}$ and Supplementary Figure S1A). CCK8 assay and cell growth curve results then showed that ADAMTS9 suppressed cell proliferation and viability (Figure 2D and Supplementary Figure S1B). The results of colony formation assays were consistent with the CCK8 assay results (Figure 2E and Supplementary Figure S1C). Thus, ADAMTS9 inhibited GC cell proliferation and viability.

\section{ADAMTS9 inhibited tumor growth in nude mouse}


We investigated the effects of ADAMTS9 in vivo. The size of subcutaneous tumor transfected with ADAMTS9 was significantly decreased than that in tumor transfected with empty vector. The growth curve and tumor weight figure of the subcutaneous tumor are shown in Figure 2F. ADAMTS9 inhibited tumor growth in vivo.

\section{ADAMTS9 inhibited GC cells migration and invasion}

The effects of ADAMTS9 expression on AGS, BGC823, and SGC-7901 cells on migration and invasion were also assessed. Wound healing assay result showed that the migration distances of over-expressed ADAMTS9 group were significantly reduced compared with that of the control group (AGS, $48 \mathrm{H}, \mathrm{P}<0.001$; BGC-823, 48H, P=0.009; and SGC-7901 36H P<0.001, Figure 2G and Supplementary Figure S1D). To confirm the effect on migration and invasion, we further examined motile ability by using Transwell chambers. Similar results were obtained, thereby indicating that the number of migratory and invaded AGS and BGC-823 cells was significantly decreased in the over-expressed ADAMTS9 group (AGS, BGC-823, and SGC-7901; migration: $P=0.003, P=0.010$, and $P=0.010$; invasion: $P=0.035, P=0.010$, and $P=0.004$; Figure $2 H$ and Supplementary Figure S1E). ADAMTS9 inhibited GC cell migration and invasion.

\section{ADAMTS9 inhibited GC metastasis through CCL5 and CXCL11 pathways}

With IHC results for ADAMTS9, we found ADAMTS9 was partly located in the nucleus of the gastric tissue cells (Figure 3A). To elucidate the downstream molecular mechanism of ADAMTS9, we performed the mRNA sequencing analysis of BGC-823 cells transfected with pCDNA3.1-ADAMTS9 plasmid or pCDNA3.1 empty plasmid. Among the 20031 genes screened by the mRNA sequencing, 183 genes showed significant decrease or increase greater than 1.5-fold change (Figure 3B). Several DEGs were significantly up- or downregulated in AGS and BGC-823 cells by real-time PCR analysis (Figure 3F). The gene information and potential biological functions of these DEGs are shown in the Figure 3D and Figure 3E. To further examine the key roles of ADAMTS9 on GC metastasis, we performed the KEGG pathway analysis (Figure $3 \mathbf{C}$ ). The chemokine signaling pathway and the cytokine-cytokine receptor interaction pathway were included in the top 20 enrichment pathways (Supplementary Figure S2). Two of the significantly down-regulated genes, namely, CCL5 and CXCL11, played a crucial role in metastasis. To further validate this finding, we detected the down-regulated mRNA and protein expression of CCL5 and CXCL11 in the four GC cell lines (AGS, BGC823, SGC-7901, and NCl-N87; Figures $\mathbf{3 G}$ and $\mathbf{3 H}$ ). The relationships between ADAMTS9 and CCL5 and between ADAMTS9 and CXCL11 in human GC tissues were further explored. We performed immunohistochemical staining to examine the correlation between the protein levels of CCL5, CXCL11, and ADAMTS9 in the GC tissue specimens on TMAs (Supplementary Figure S3). Figure 3 I shows that the protein expressions of CCL5 and CXCL11 were both negatively associated with ADAMTS9 protein expression (CCL5, $N=122$, Pearson $r=-0.308, P=0.001$; $C X C L 11, N=125$, Pearson $r=-0.501, P<0.001$ ). ADAMTS9 inhibited the GC cell metastasis by down-regulating CCL5 and CXCL11 expressions. 


\section{DNMT3A mainly regulated the promotor methylation of ADAMTS9 gene}

According to the MethHC database(http://methhc.mbc.nctu.edu.tw/php), the ADAMTS9 promotor is hypermethylated in the GC, thereby leading to the down-regulation or silencing of the expression of ADAMTS9 (Figure 4A). We also validated the hypermethylation of ADAMTS9 promotor by using the 5-Aza $(2 \mu \mathrm{M})$ treatment in the AGS and BGC-823 cell lines. Figure 4B shows that the mRNA expression of ADAMTS9 significantly increased after 5-Aza $(2 \mu \mathrm{M})$ treatment. MassARRAY analysis was adopted to examine the methylation of ADAMTS9 DNA promotor. Figure 4C and Supplementary Figure S4A show that aberrant methylation was detected in BGC823 cells treated with 5-Aza (2 $\mu \mathrm{M})$. In human tissues, DNMT1, DNMT3A, and DNMT3B are the most common methyltransferases that play key roles in various biological functions. We then explored the mechanism underlying the regulation of promotor methylation of ADAMTS9 DNA. AGS and BGC-823 cell lines were transfected with shDNMT1, shDNMT3A, or shDNMT3B (Supplementary Figure S5). PCR and Western blot analysis results showed that the DNMT3A should be the main methyltransferase that regulate ADAMTS9 expression (Figure 4D). DNA methylation analysis through MassARRAY platform also confirmed that downregulated DNMT1 and DNMT3A expressions significantly increased the methylation level of ADAMTS9 gene in the BGC823 cell, especially for DNMT3A (Figure 4Eand Supplementary Figures S4B and S4C).

\section{RNF180 restores ADAMTS9 expression by promoting DNMT3A ubiquitination and degradation}

Our previous studies reported RNF180 plays the tumor suppressive roles on patients with GC. In this study, the IHC results of TMAs showed a significant positive correlation between RNF180 and ADAMTS9 expression.( $\mathrm{N}=126$, Pearson $\mathrm{r}=0.454, \mathrm{P}<0.001$, Figure 5A and Supplementary Figure S6). In addition, with the correlation analysis from the GEPIA database (http://gepia.cancer-pku.cn/), we found that the mRNA expression level of RNF180 might be positively correlated with that ADAMTS9 (Spearman $r=0.28, P=5 e-09$, Pearson $r=0.18, P=0.00024$, Figure 5B) Therefore, we hypothesized that the RNF180 restores ADAMTS9expression by decreasing ADAMTS9 methylation. Figure $\mathbf{5 C}$ shows the results of PCR and Western blot analysis, which indicated that RNF180 upregulated ADAMTS9 expression through the epigenetic pathway. We then performed the methylation analysis of ADAMTS9 in the BGC823 cells transfected with RNF180, and the significant downregulated methylation of ADAMTS9 DNA was detected (Figure 5Dand Supplementary Figure S4D). These results confirmed our previous hypothesis.

Considering that RNF180 restores the expression of ADAMTS9 by decreasing the methylation of ADAMTS9 promotor and that the methylation of ADAMTS9 promotor is mainly regulated by DNMT3A, we hypothesized that RNF180 ubiquitinated DNMT3A, which promoted their degradation via proteasome pathway. The CHX pulse-chase assay was performed to examine the function of RNF180 on DNMT3A stability. We observed that RNF180 promoted the degradation of DNMT3A protein and reduced their halflife. (Figure 5E). AGS and BGC823 cells were then incubated with MG132 (10 $\mu \mathrm{M})$ to inhibit protein 
degradation via proteasome pathway. Figure $5 \mathrm{~F}$ shows that RNF180 promoted the accumulation of DNMT3A after treatment with MG132. Hence, we speculated that DNMT3A might be the substrate of RNF180. To explore the interaction between RNF180 and DNMT3A, we performed the coimmunoprecipitation assay. The reciprocal co-immunoprecipitation confirmed the interaction between DNMT3A and RNF180 (Figure 5G). Ubiquitination assay was also performed to validate that the ubiquitination level of DNMT3A was greatly promoted by RNF180 (Figure $5 \mathrm{H}$ ). In conclusion, ADAMTS9 was restored by RNF180 via promoting DNMT3A ubiquitination and degradation.

\section{Discussion:}

LN metastasis is a crucial factor to predict the survival outcomes, and the great understanding of the LN metastasis benefits how clinicians treat patients with GC. According to previous studies, ADAMTS9 is a tumor suppressor that inhibits the proliferation of several types of malignant tumors, such as esophageal ${ }^{8}$, and breast cancers ${ }^{10}, \mathrm{GC}^{12}$, and nasopharyngeal carcinoma ${ }^{9}$. However, the involvement of ADAMTS9 in LN metastasis and as the predictive factor in GC have been rarely reported. In this study, we provided experimental and clinical evidences to support the suppressive effect of ADAMTS9 on LN metastasis in GC. Our study demonstrated that ADAMTS9 inhibited migration and invasion in GC cells. Clinical data suggested that the low expression of ADAMTS9 was closely associated with advanced $\mathrm{pN}$ stage and poor survival outcomes, which was identified as an independent risk factor. ADAMTS9 might be a potential biomarker to predict the risk of $L N$ metastasis and improve the understanding of $L N$ metastasis mechanism in GC.

The hypermethylation of $\mathrm{CpG}$ islands in the gene promotor region of ADAMTS9, which was detected by the MassARRAY analysis, was closely associated with the absent or down-regulated ADAMTS9 expression. And ADAMTS9 expression was largely regulated by the promotor methylation. These findings were consistent with those obtained in previous study ${ }^{12}$. Silencing or promoting some tumor-related genes through hypermethylation or hypomethylation plays a crucial role in the occurrence and development of various cancer ${ }^{17}$. Thus, the potent molecular mechanism of the promotor methylation of ADAMTS9 must be investigated. In this study, ADAMTS9 expression was mostly inhibited by DNMT3A by enhancing the promotor methylation. DNMT1, DNMT3A, and DNMT3B are the three catalytic active DNMTs in mammals, which participate in a diverse range of biological processes ${ }^{18}$. DNMT3A and DNMT3B are responsible for the maintenance of DNA methylation through de novo methylation activity in the early stage of embryo or during cell differentiation, whereas DNMT1 is mostly responsible for the maintenance of DNA methylation during replication ${ }^{19}$. The disorder in the expression of DNMTs can elicit the hypo- or hyper-methylation of several tumor-related genes through epigenetic changes ${ }^{20-22}$. Through the online GEPIA database and IHC staining in this study, we found a significant positive correlation between the expression of RNF180 and ADAMTS9. According to our previous studies, RNF180, which is an E3 ubiquitin ligase, is a suppressor gene that inhibits LN metastasis ${ }^{14}$. RNF180 belongs to the ubiquitin-proteasome system that plays an important role in several oncogenesis and tumor progression processes ${ }^{23}$, whereas the substrate of RNF180 remains unclear. In present study, we uncovered that RNF180 could reduce the stability of 
DNMT3A through the ubiquitination process, and that DNMT3A was identified as the substrate of RNF180. In such a process, RNF180, as a tumor suppressor, could restore some tumor-related genes, including ADAMTS9, and played a crucial role in impairing the lymphatic involvement of GC cells. In conclusion, ADAMTS9 was restored by RNF180-mediated ubiquitination and degradation of DNMT3A.

We further investigated the effect of ADAMTS9 on GC metastasis and its downstream molecular pathway. ADAMTS9 inhibited GC metastasis, mostly by regulating chemokine signaling pathway and cytokinecytokine receptor interaction pathway, which were included in the top 20 KEGG enrichment pathways (SupplementaryFigure S2). Infection and chronic inflammation can promote tumor development in a tumor microenvironment. This phenomenon has become the hallmark of cancer, recently ${ }^{2425}$. The recruited stomal or inflammatory cells by tumor cells or by "educated" non-tumor cells through direct interaction or indirect signals (several cytokines and chemokines) constitute the tumor microenvironment ${ }^{26}$. Chemokines is an important component of the tumor microenvironment and plays an important role in inflammation and also in cancer development ${ }^{27}$. In this study, we demonstrated that ADAMTS9 inhibited LN metastasis in GC, through CCL5- and CXCL11-dependent pathways. First, CCL5/CCR5 axis is closely correlated with tumor metastasis ${ }^{2829}$. The high CCL5 or CCR5 expression in patients with GC were associated with a poor survival rate ${ }^{30}$ and increased metastasis risk ${ }^{31}$. CCL 5 could activate the av 33 integrin through PI3K/Akt pathway, causing the upregulation of IKK alpha/beta and NF$\mathrm{KB}^{32}$, which eventually induced the secretion of MMP-933 or upregulated the secretion of both MMP-9 and MMP- $2^{34}$. CCL5 contributed to tumor cell metastasis by degrading the extracellular matrix. In addition, W Du et al. offered the indirect evidence that ADAMTS9 plays a tumor-suppressive effect through Akt pathway ${ }^{12}$, which is mostly consistent with the downstream pathway of CCL5. The CCL5/CCR5 axis was the important intermediate element of ADAMTS9's inhibition of the GC cell invasion and metastasis. Second, CXCL11 can increase the metastatic behavior of cancer cells in colorectal ${ }^{35}$, ovarian ${ }^{36}$, and prostate cancers ${ }^{37}$. The mechanism of $\mathrm{CXCL} 11$ in promoting metastasis was based on the chemokine receptors $\mathrm{CXCR} 3$ and $\mathrm{CXCR} 7^{38}$. The mutual interaction of $\mathrm{CXCR} 3$ system and $\mathrm{CXCR7}$ system formed a complex functional network ${ }^{39}$. Even though CXCR3 and CXCR7 systems both regulate tumor growth and metastasis in various types of cancer ${ }^{40}$, the explicit mechanism of CXCL11 currently remains unclear and must be further investigated. Nevertheless, CXCL11-CXCR3/CXCR7 axis can clearly contribute to tumor cell migration and invasion.

\section{Conclusions:}

In conclusion, the low ADAMTS9 expression was closely associated with advanced pN stage and poor survival outcome. Low ADAMTS9 expression might be a biomarker to predict the metastatic condition in patients with GC. ADAMTS9 expression was restoredby RNF180 via promoting DNMT3A ubiquitination and degradation. ADAMTS9 inhibited GC cell metastasis by inactivating CCL5- and CXCL11-dependent pathways.

\section{Limitations:}


This study contains several potential limitations. First, the TMAs used in this study enrolled the GC tissues and adjacent non-tumor tissues between August 2004 and December 2007. Although TMAs were stored properly in $-4{ }^{\circ} \mathrm{C}$, some specimens were excluded, which might have caused evaluation bias. Second, the potential pathways of CCL5 and CXCL11 in various types of cancer have been extensively reported. In this study, we did not validate the downstream molecular mechanisms of CCL5 and CXCL11.

\section{List Of Abbreviations:}

ADAMTS9, a disintegrin-like and metalloprotease with thrombospondin type 1 motif 9; GC, gastric cancer; LN, lymph node; CCL5, C-C motif chemokine ligand 5; CXCL11, C-X-C motif chemokine ligand 11; DNMT, DNA-methyltransferase; RNF, Ring finger protein; TMA, tissue microarray; IHC, immunohistochemistry; $\mathrm{CHX}$, cycloheximide; TCGA, The Cancer Genome Atlas; OS, overall survival; HR, hazard ratios; $95 \% \mathrm{Cl}, 95 \%$ confidence interval; DEG, differentially expressed genes.

\section{Declarations:}

\section{Ethics approval and consent to participate}

The study protocol and permission for the use of the clinical data and human tissue were given by the Institutional Research Ethics Committee of Tianjin Medical University Cancer Institute and Hospital (Tianjin, China).

\section{Consent for publication}

Not applicable.

\section{Availability of data and materials}

The datasets used and/or analysed during the current study are available from the corresponding author on reasonable request.

\section{Competing interests}

The authors declare that they have no competing interests.

\section{Funding}

This study was supported in part by grants from National Key Research and Development Program "precision medicine research" (2017YFC0908304), the Programs of National Natural Science Foundation of China (NO. 81572372), , National Key Research and Development Program "major chronic non- 
infectious disease research" (2016YFC1303202), and the Natural Science Foundation of China (NO.81602153).

\section{Authors' contributions}

Jingyu Deng, Gang Ma, and Han Liang designed the experiments;

Weilin Sun, Gang Ma, Li Zhang, Pengliang Wang and Fenglin Cai conducted the experiments;

Weilin Sun, Huifang Liu, Nannan Zhang and Zizhen Wu Analyzed the data and prepared the figures;

Weilin Sun, Yinping Dong, Liqiao Chen and Zhenzhen Zhao wrote the manuscript;

Wei-lin Sun, Jing-yu Deng and Gang Ma equally contributed to the work.

The manuscript got the writing assistance from EssaySta (https://essaystar.com/) website.

\section{Acknowledgements}

The manuscript got the writing assistance from EssayStar(https://essaystar.com/) website.

\section{References:}

1. Bray F, Ferlay J, Soerjomataram I, et al. Global cancer statistics 2018: GLOBOCAN estimates of incidence and mortality worldwide for 36 cancers in 185 countries. CA: a cancer journal for clinicians 2018;68(6):394-424. doi: 10.3322/caac.21492

2. Karimi P, Islami F, Anandasabapathy S, et al. Gastric cancer: descriptive epidemiology, risk factors, screening, and prevention. Cancer epidemiology, biomarkers \& prevention : a publication of the American Association for Cancer Research, cosponsored by the American Society of Preventive Oncology 2014;23(5):700-13. doi: 10.1158/1055-9965.EPI-13-1057

3. Clark ME, Kelner GS, Turbeville LA, et al. ADAMTS9, a novel member of the ADAM-TS/ metallospondin gene family. Genomics 2000;67(3):343-50. doi: 10.1006/geno.2000.6246

4. Ozler S, Isci Bostanci E, Oztas E, et al. The role of ADAMTS4 and ADAMTS9 in cardiovascular disease in premature ovarian insufficiency and idiopathic hypogonadotropic hypogonadism. Journal of endocrinological investigation 2018;41(12):1477-83. doi: 10.1007/s40618-018-0948-3

5. Kumagishi K, Nishida K, Yamaai T, et al. A disintegrin and metalloproteinase with thrombospondin motifs 9 (ADAMTS9) expression by chondrocytes during endochondral ossification. Archives of histology and cytology 2009;72(3):175-85. doi: 10.1679/aohc.72.175

6. Lo PH, Lung HL, Cheung AK, et al. Extracellular protease ADAMTS9 suppresses esophageal and nasopharyngeal carcinoma tumor formation by inhibiting angiogenesis. Cancer research 2010;70(13):5567-76. doi: 10.1158/0008-5472.CAN-09-4510 
7. Koo BH, Coe DM, Dixon LJ, et al. ADAMTS9 is a cell-autonomously acting, anti-angiogenic metalloprotease expressed by microvascular endothelial cells. The American journal of pathology 2010;176(3):1494-504. doi: 10.2353/ajpath.2010.090655

8. Lo PH, Leung AC, Kwok CY, et al. Identification of a tumor suppressive critical region mapping to 3p14.2 in esophageal squamous cell carcinoma and studies of a candidate tumor suppressor gene, ADAMTS9. Oncogene 2007;26(1):148-57. doi: 10.1038/sj.onc.1209767

9. Lung HL, Lo PHY, Xie D, et al. Characterization of a novel epigenetically-silenced, growth-suppressive gene, ADAMTS9, and its association with lymph node metastases in nasopharyngeal carcinoma. International journal of cancer 2008;123(2):401-08. doi: 10.1002/ijc.23528

10. Shao B, Feng $Y$, Zhang $H$, et al. The 3p14.2 tumour suppressor ADAMTS9 is inactivated by promoter $\mathrm{CpG}$ methylation and inhibits tumour cell growth in breast cancer. Journal of cellular and molecular medicine 2018;22(2):1257-71. doi: 10.1111/jcmm.13404

11. Chen L, Tang J, Feng Y, et al. ADAMTS9 is Silenced by Epigenetic Disruption in Colorectal Cancer and Inhibits Cell Growth and Metastasis by Regulating Akt/p53 Signaling. Cellular physiology and biochemistry : international journal of experimental cellular physiology, biochemistry, and pharmacology 2017;44(4):1370-80. doi: 10.1159/000485534

12. Du W, Wang $S$, Zhou Q, et al. ADAMTS9 is a functional tumor suppressor through inhibiting AKT/mTOR pathway and associated with poor survival in gastric cancer. Oncogene 2013;32(28):3319-28. doi: 10.1038/onc.2012.359

13. Urnov FD. Methylation and the genome: the power of a small amendment. The Journal of nutrition 2002;132(8 Suppl):2450S-56S. doi: 10.1093/jn/132.8.2450S

14. Deng J, Liang $\mathrm{H}$, Zhang R, et al. Clinical and experimental role of ring finger protein 180 on lymph node metastasis and survival in gastric cancer. The British journal of surgery 2016;103(4):407-16. doi: 10.1002/bjs. 10066

15. Popovic D, Vucic D, Dikic I. Ubiquitination in disease pathogenesis and treatment. Nature medicine 2014;20(11):1242-53. doi: 10.1038/nm.3739

16. Kanehisa M, Araki M, Goto S, et al. KEGG for linking genomes to life and the environment. Nucleic acids research 2008;36(Database issue):D480-4. doi: 10.1093/nar/gkm882

17. Kulis M, Esteller M. DNA methylation and cancer. Advances in genetics 2010;70:27-56. doi: 10.1016/B978-0-12-380866-0.60002-2

18. Morgan AE, Davies TJ, Mc Auley MT. The role of DNA methylation in ageing and cancer. The Proceedings of the Nutrition Society 2018;77(4):412-22. doi: 10.1017/S0029665118000150

19. Fattahi S, Golpour M, Amjadi-Moheb F, et al. DNA methyltransferases and gastric cancer: insight into targeted therapy. Epigenomics 2018;10(11):1477-97. doi: 10.2217/epi-2018-0096

20. Gao Q, Steine EJ, Barrasa MI, et al. Deletion of the de novo DNA methyltransferase Dnmt3a promotes lung tumor progression. Proceedings of the National Academy of Sciences of the United States of America 2011;108(44):18061-6. doi: 10.1073/pnas.1114946108 
21. Fu HY, Shen JZ, Wu Y, et al. Arsenic trioxide inhibits DNA methyltransferase and restores expression of methylation-silenced CDKN2B/CDKN2A genes in human hematologic malignant cells. Oncology reports 2010;24(2):335-43. doi: 10.3892/or_00000864

22. Zheng Y, Huang Q, Ding Z, et al. Genome-wide DNA methylation analysis identifies candidate epigenetic markers and drivers of hepatocellular carcinoma. Briefings in bioinformatics 2018;19(1):101-08. doi: 10.1093/bib/bbw094

23. Cheung KF, Lam CN, Wu K, et al. Characterization of the gene structure, functional significance, and clinical application of RNF180, a novel gene in gastric cancer. Cancer 2012;118(4):947-59. doi: 10.1002/cncr.26189

24. Bottcher JP, Bonavita E, Chakravarty P, et al. NK Cells Stimulate Recruitment of cDC1 into the Tumor Microenvironment Promoting Cancer Immune Control. Cell 2018;172(5):1022-37 e14. doi: 10.1016/j.cell.2018.01.004

25. Halama N, Zoernig I, Berthel A, et al. Tumoral Immune Cell Exploitation in Colorectal Cancer Metastases Can Be Targeted Effectively by Anti-CCR5 Therapy in Cancer Patients. Cancer cell 2016;29(4):587-601. doi: 10.1016/j.ccell.2016.03.005

26. Yang L, Lin PC. Mechanisms that drive inflammatory tumor microenvironment, tumor heterogeneity, and metastatic progression. Seminars in cancer biology 2017;47:185-95. doi: 10.1016/j.semcancer.2017.08.001

27. Chow MT, Luster AD. Chemokines in cancer. Cancer immunology research 2014;2(12):1125-31. doi: 10.1158/2326-6066.CIR-14-0160

28. Zhang Q, Qin J, Zhong L, et al. CCL5-Mediated Th2 Immune Polarization Promotes Metastasis in Luminal Breast Cancer. Cancer research 2015;75(20):4312-21. doi: 10.1158/0008-5472.CAN-14-3590

29. Aldinucci D, Casagrande N. Inhibition of the CCL5/CCR5 Axis against the Progression of Gastric Cancer. International journal of molecular sciences 2018;19(5) doi: 10.3390/ijms19051477

30. Sugasawa $\mathrm{H}$, Ichikura $\mathrm{T}$, Tsujimoto $\mathrm{H}$, et al. Prognostic significance of expression of CCL5/RANTES receptors in patients with gastric cancer. Journal of surgical oncology 2008;97(5):445-50. doi: $10.1002 /$ jso.20984

31. Wang T, Wei Y, Tian L, et al. C-C motif chemokine ligand 5 (CCL5) levels in gastric cancer patient sera predict occult peritoneal metastasis and a poorer prognosis. International journal of surgery 2016;32:136-42. doi: 10.1016/j.ijsu.2016.07.008

32. Huang CY, Fong YC, Lee CY, et al. CCL5 increases lung cancer migration via PI3K, Akt and NF-kappaB pathways. Biochemical pharmacology 2009;77(5):794-803. doi: 10.1016/j.bcp.2008.11.014

33. Long $\mathrm{H}$, Xie R, Xiang $\mathrm{T}$, et al. Autocrine CCL5 signaling promotes invasion and migration of CD133+ ovarian cancer stem-like cells via NF-kappaB-mediated MMP-9 upregulation. Stem cells 2012;30(10):2309-19. doi: 10.1002/stem.1194

34. Kato T, Fujita Y, Nakane K, et al. CCR1/CCL5 interaction promotes invasion of taxane-resistant PC3 prostate cancer cells by increasing secretion of MMPs $2 / 9$ and by activating ERK and Rac signaling. Cytokine 2013;64(1):251-7. doi: 10.1016/j.cyto.2013.06.313 
35. Gao YJ, Liu L, Li S, et al. Down-regulation of CXCL11 inhibits colorectal cancer cell growth and epithelial-mesenchymal transition. OncoTargets and therapy 2018;11:7333-43. doi: 10.2147/OTT.S167872

36. Koo YJ, Kim TJ, Min KJ, et al. CXCL11 mediates TWIST1-induced angiogenesis in epithelial ovarian cancer. Tumour biology : the journal of the International Society for Oncodevelopmental Biology and Medicine 2017;39(5):1010428317706226. doi: 10.1177/1010428317706226

37. Wang Y, Xu H, Si L, et al. MiR-206 inhibits proliferation and migration of prostate cancer cells by targeting CXCL11. The Prostate 2018;78(7):479-90. doi: 10.1002/pros.23468

38. Puchert M, Obst J, Koch $\mathrm{C}$, et al. CXCL11 promotes tumor progression by the biased use of the chemokine receptors CXCR3 and CXCR7. Cytokine 2020;125:154809. doi:

10.1016/j.cyto.2019.154809

39. Singh AK, Arya RK, Trivedi AK, et al. Chemokine receptor trio: CXCR3, CXCR4 and CXCR7 crosstalk via CXCL11 and CXCL12. Cytokine \& growth factor reviews 2013;24(1):41-9. doi: 10.1016/j.cytogfr.2012.08.007

40. Freitas C, Desnoyer A, Meuris F, et al. The relevance of the chemokine receptor ACKR3/CXCR7 on CXCL12-mediated effects in cancers with a focus on virus-related cancers. Cytokine \& growth factor reviews 2014;25(3):307-16. doi: 10.1016/j.cytogfr.2014.04.006

\section{Figures}


A

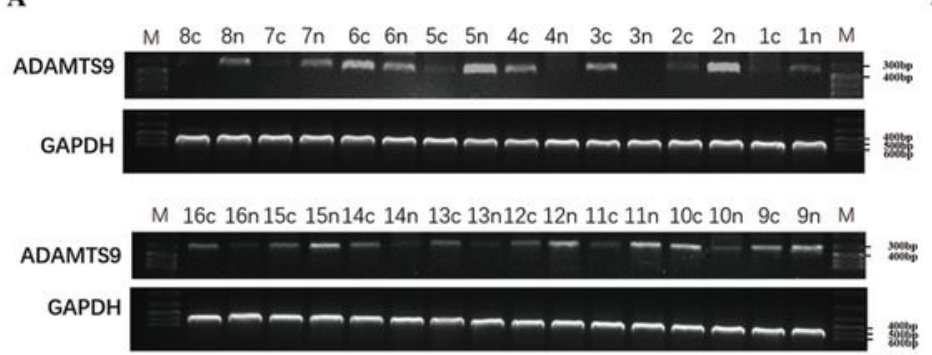

C, gastric cancer tissue; $N$, matched non-tumor tissue; $M$, marker
B

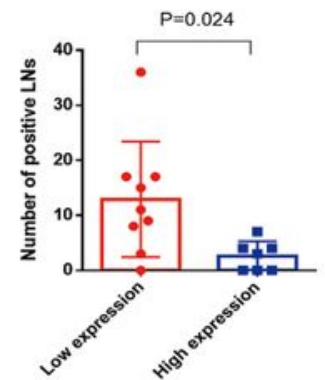

C

Gastric cancer tissue
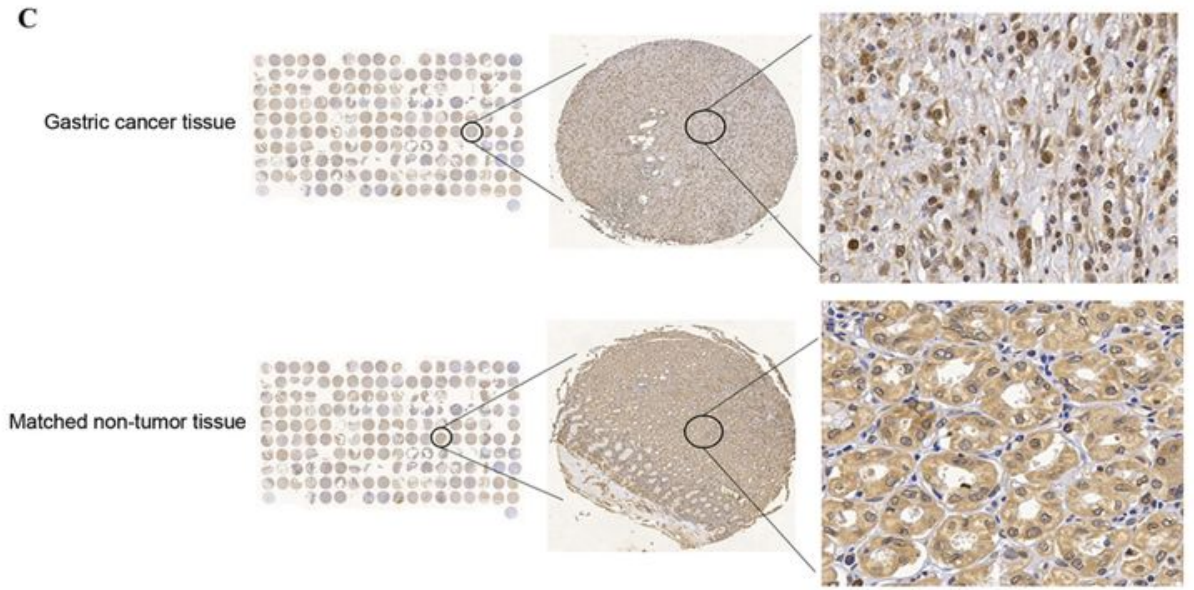

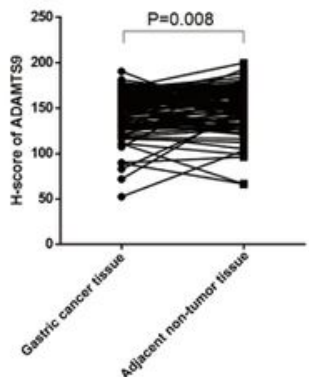

$\mathbf{E}$

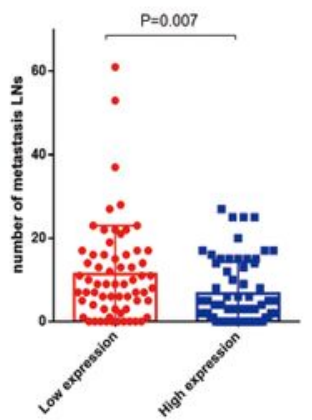

$\mathbf{F}$
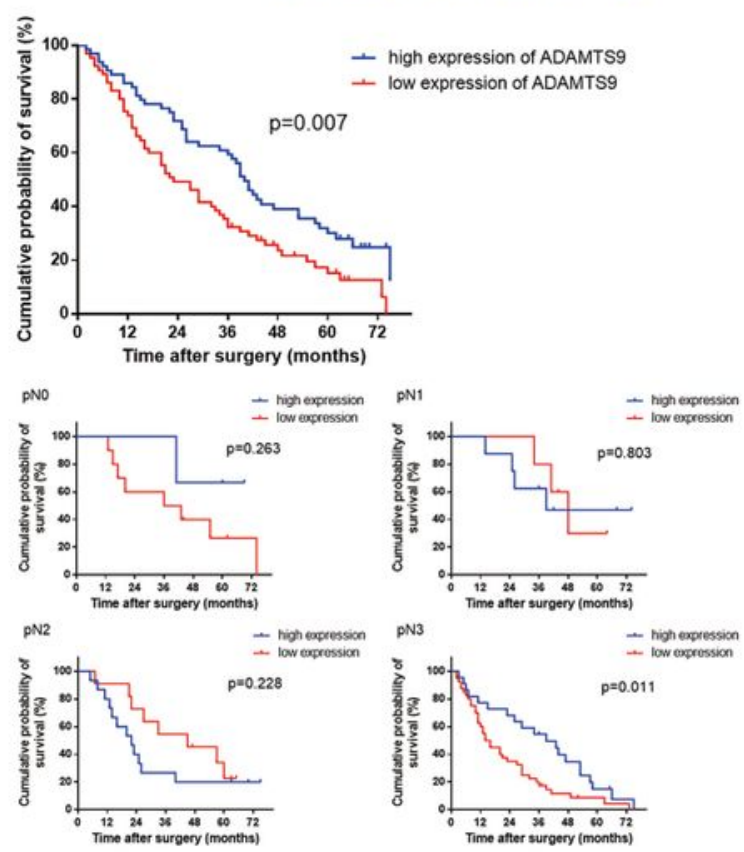

Figure 1 Low expression of ADAMTS9 in GC is associated with more metastatic lymph nodes and poorer survival outcomes.

\section{Figure 1}

Low expression of ADAMTS9 in GC is associated with more metastatic lymph nodes and poorer survival outcomes. (A) mRNA expression levels of ADAMTS9 in GC tissues and matched non-tumor tissues. (B) Association between ADAMTS9 mRNA expression and metastatic lymph nodes. (C) IHC of ADAMTS9 in GC tissues and matched adjacent non-tumor tissues. (D) Low expression of ADAMTS9 protein in GC 
tissues. (E) Association between the expression of ADAMTS9 protein and metastatic lymph nodes. (F) Keplan Meier survival analysis in 129 GC patients and stratification by pN stage.

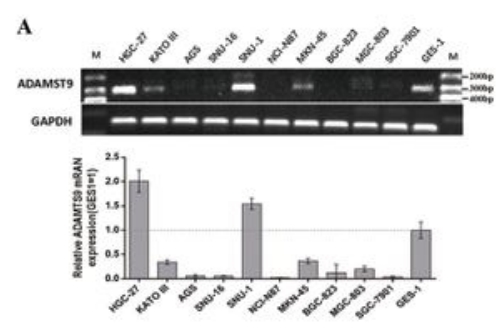

C

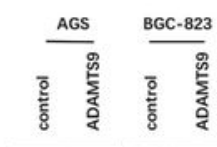

ADAMTS9

GAPDH

ADAMTS9

$\beta$-actin
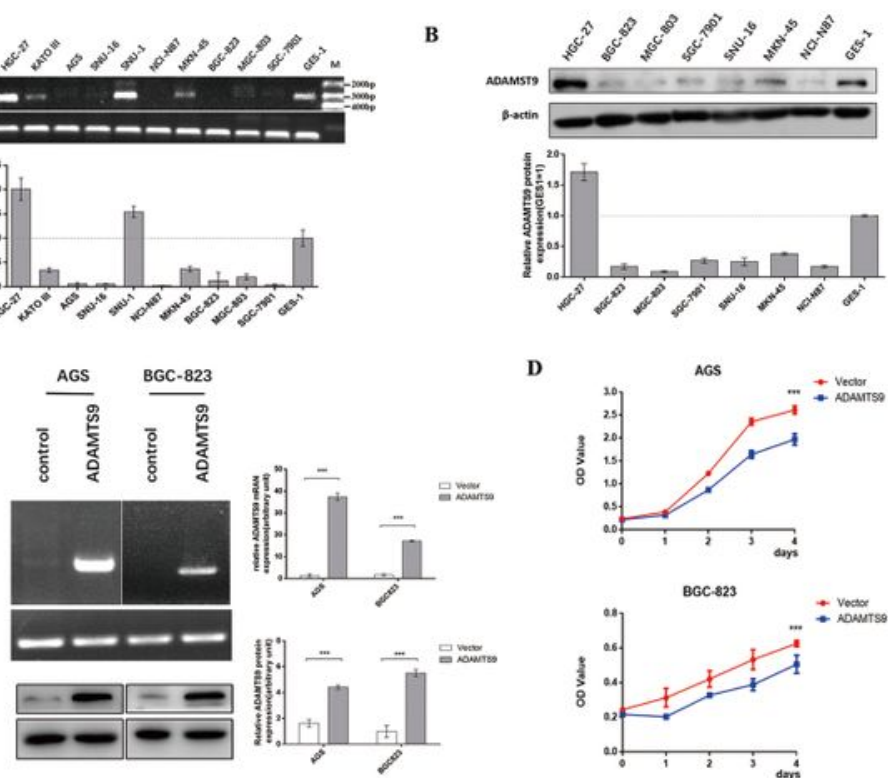

D
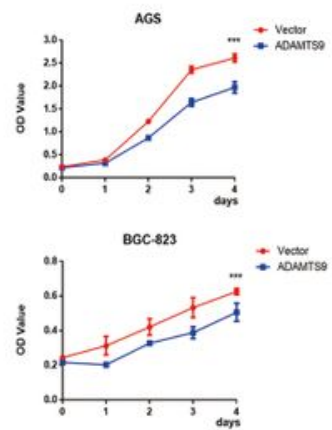

E
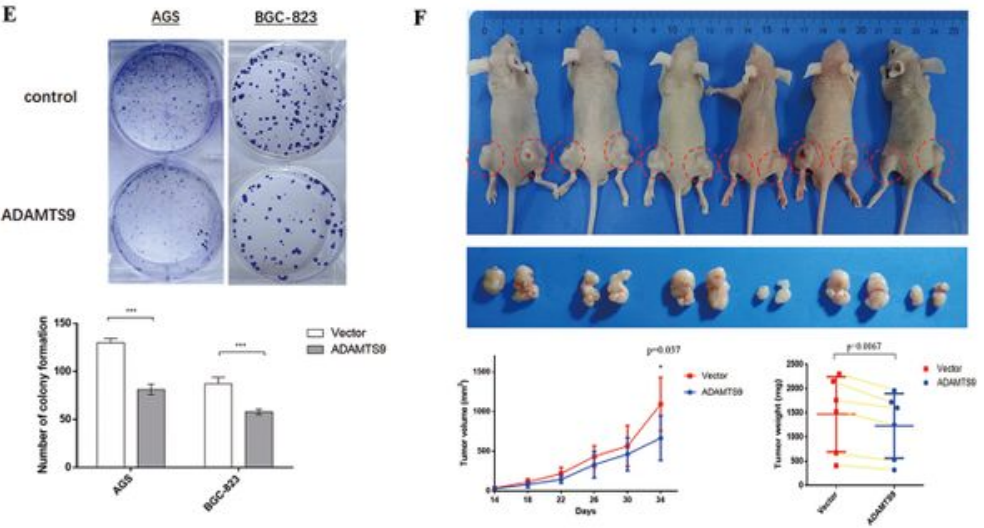

G

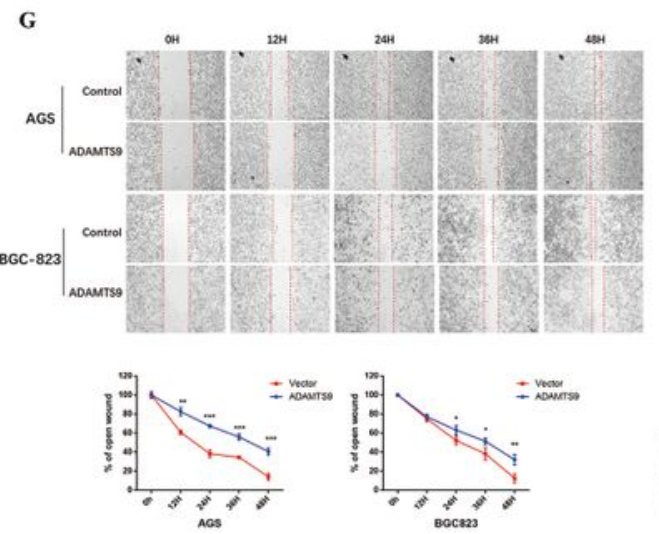

\begin{abstract}
H
\end{abstract}

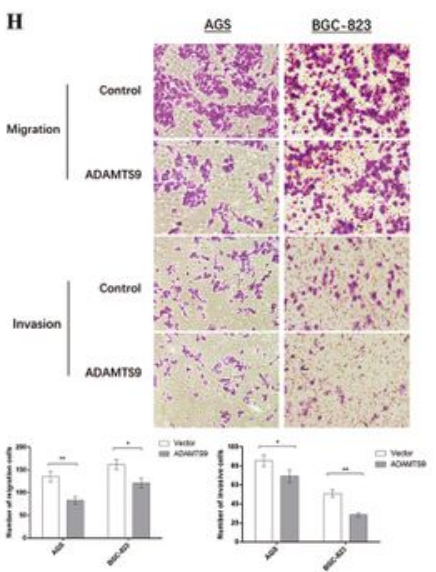

Figure 2 The ADAMTS9 expression in GC cell lines and biology function of ADAMTS9 in GC

\section{Figure 2}

The ADAMTS9 expression in GC cell lines and biology function of ADAMTS9 in GC. (A) mRNA expression levels of ADMATS9 in ten GC cell lines and normal human gastric epithelial cell line (GES-1). (B) Western blotting of protein from seven GC cell lines and normal human gastric epithelial cell line (GES-1) for 
ADAMTS9 expression. (C) mRNA and protein expression levels of ADAMTS9 in AGS and BGC-823 cells transfected with ADAMTS9 overexpression plasmid and empty plasmid. (D) ADAMTS9 inhibits the cell growth in AGS and BGC-823 cells. (E) ADAMTS9 suppresses the colony formation in AGS and BGC-823 cells. (F) ADAMTS9 inhibited growth of tumors in vivo. Tumor growth curves and tumor weight shows the suppressive effect of ADAMTS9 in vivo.(G)ADAMTS9 impairs motile capacity in AGS and BGC-823 cells detected by wound healing assay; $(\mathrm{H})$ and by transwell assay.

A
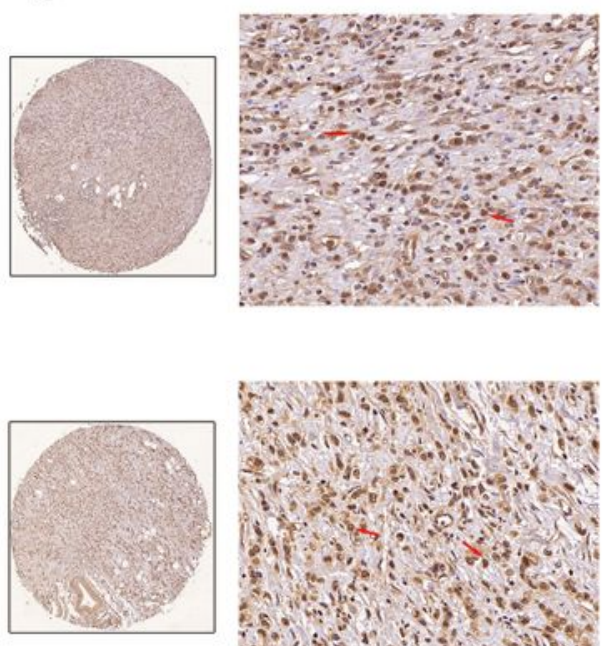

D

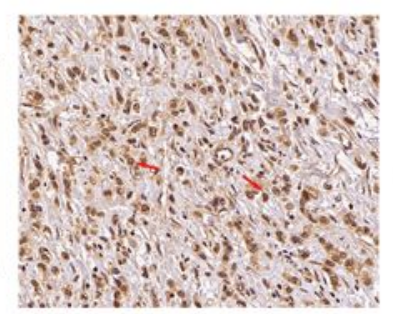

B



C

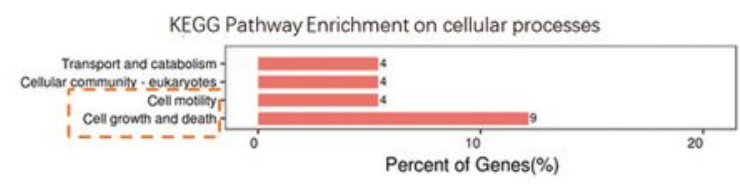

E

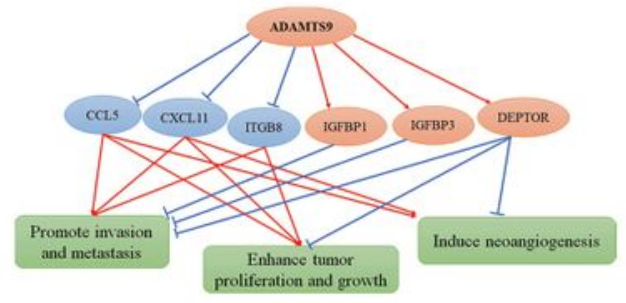

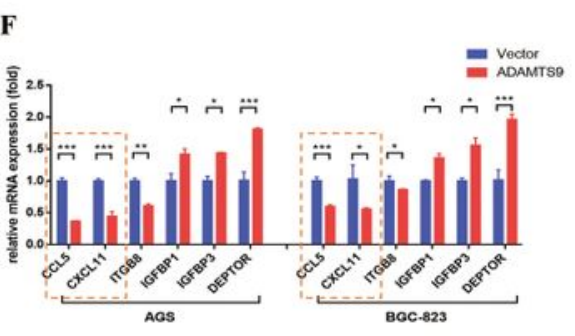

G

H



I
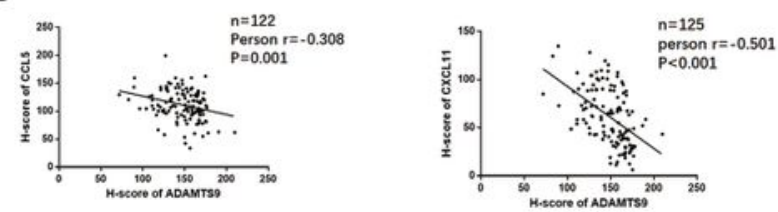

Figure 3 ADAMTS9 downregulates the expression of CCL5 and CXCL11.

\section{Figure 3}


ADAMTS9 downregulates the expression of CCL5 and CXCL11. (A) ADAMTS9 is partly located in the nucleus of the gastric tissue cells. (B) The heatmap was generated from mRNA sequencing analysis of ADAMTS9-overexpressing BGC-823 cells and control cells transfected with empty plasmid. (C) KEGG analysis of DEGs on cellular processes. (D)The foldchange and P-value of downstream potent molecular. (E)Downstream potent molecular event for impairing viability, proliferation and motile capacity. $(F)$ The downstream DEGs were confirmed by the qPCR in AGS and BGC-823 cells. (G) ADAMTS9 also inhibits transcription of CCL5 and CXCL11 in other two gastric cell lines, SGC-7901 and NCI-N87 cells, detected by qPCR; (H) ADAMTS9 downregulates the protein expression of CCL5 and CXCL11 in four gastric cell lines, detected by western blotting method. (I) IHC of TMAs shows a significant negative association between ADAMTS9 and CCL5, and between ADAMTS9 and CXCL11. 
A

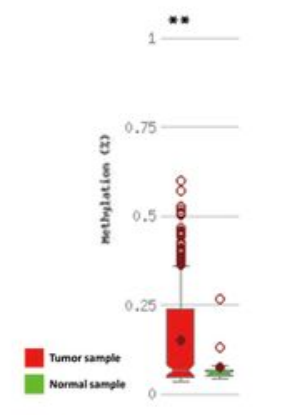

B

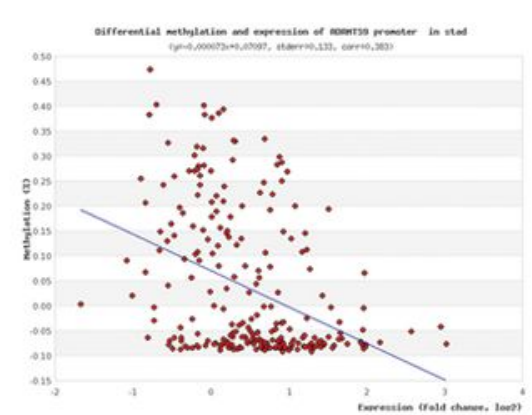

C
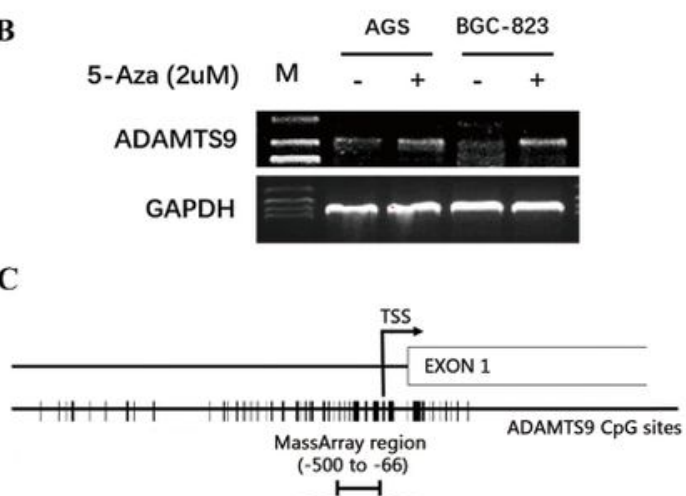

CpGno. 1234567891011121314151617
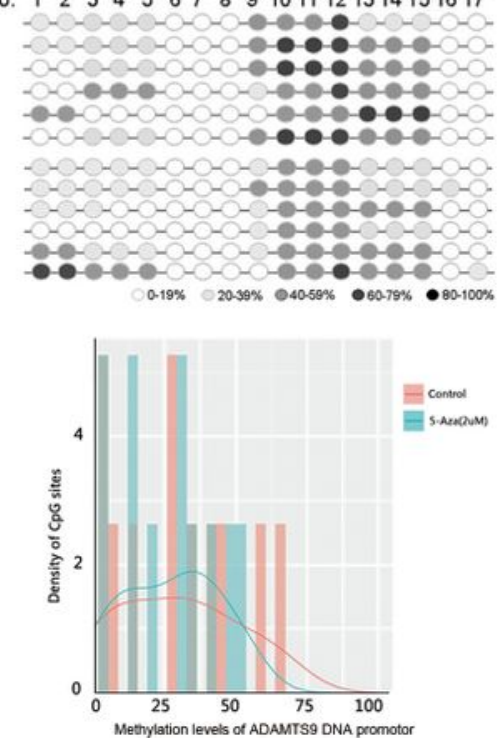

$\mathbf{E}$
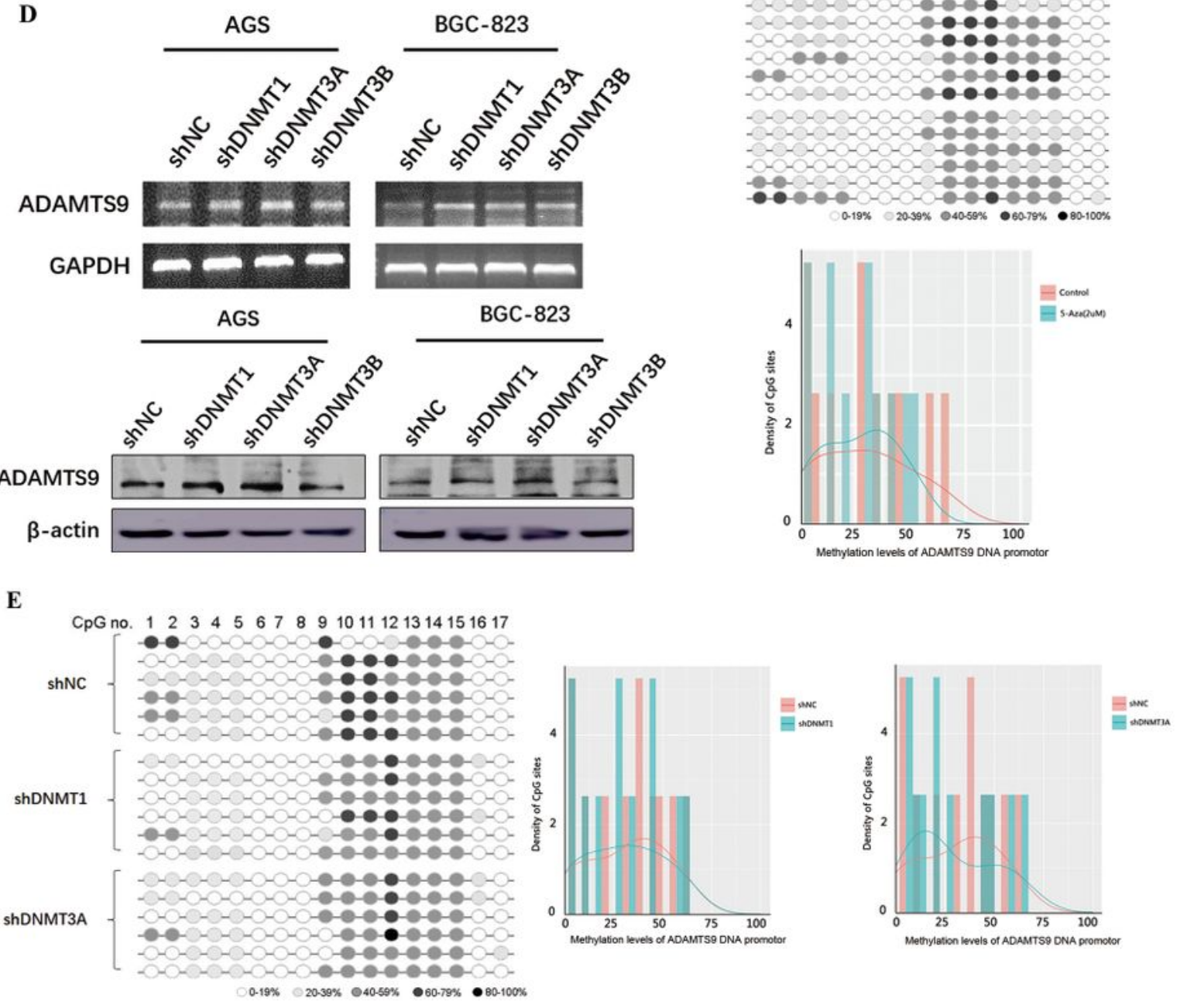

Figure 4 Hypermethylated ADAMTS9 in gastric cancer is mainly associated with DNMT3A

\section{Figure 4}

Hypermethylated ADAMTS9 in gastric cancer is mainly associated with DNMT3A. (A) ADAMTS9 DNA promotor is hypermethylated in the gastric cancer, according to the MethHC database. (B)The ADAMTS9 expression in AGS and BGC-823 cells after pharmacological reversal of DNA methylation by 5-Aza was examined by reverse transcriptase PCR. (C)The MassARRAY analysis spans the promoter region from -500 to -66, including $17 \mathrm{CpG}$ islands. The methylation levels of ADAMTS9 DNA promotor after 5-Aza 
incubation were showed. (D) The expression of ADAMTS9 in AGS and BGC-823 cells was examined by reverse transcriptase PCR and western blotting method, after transfection with shDNMT1, shDNMT3A, shDNMT3B or empty plasmid. (E)The methylation levels of ADAMTS9 DNA promotor after transfection were detected by the MassARRAY analysis.

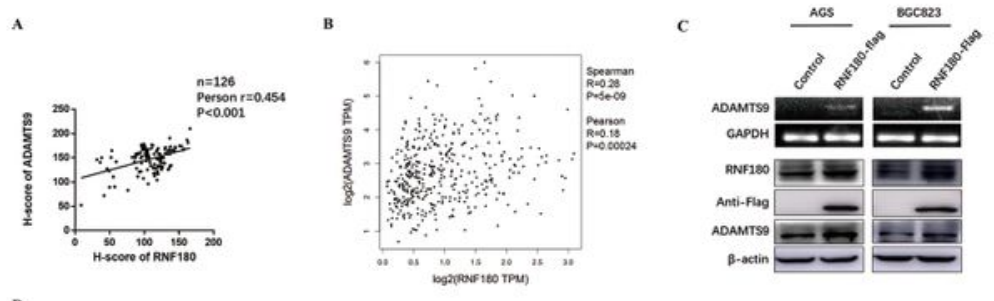

D
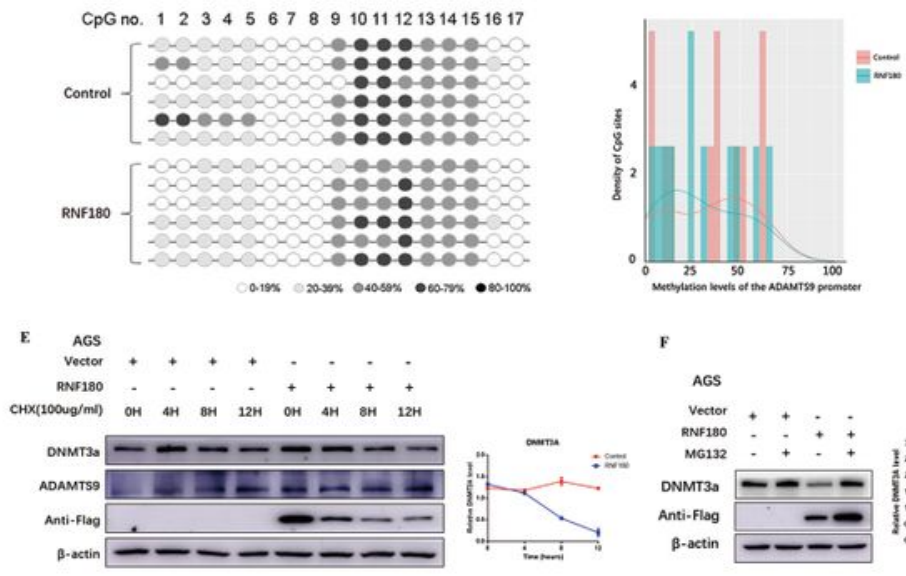

$\mathbf{F}$

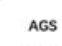

AGS
Vector
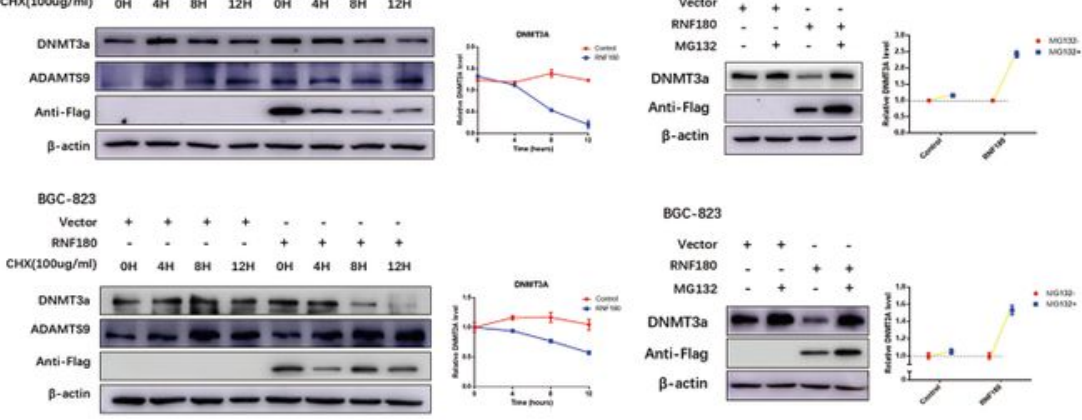

BGC-823

vector + + -

${ }_{\text {MGF132 }:+;}^{+}+$

M6132 - + + fin , :

$\beta$-actin

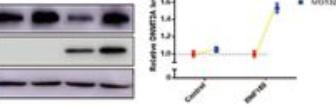

RNF180-MYC - - +

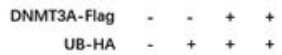

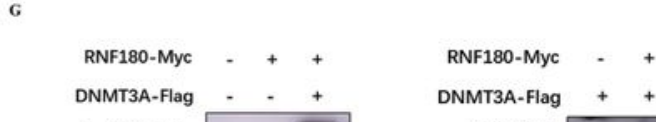

IP: Flag | $\begin{aligned} & \text { Anti-Flag } \\ & \text { Anti-Myc }\end{aligned}$ IP: Myc

$\left.\right|_{\text {Anti-Myc }} ^{\text {Anti-Flag }}$

Input

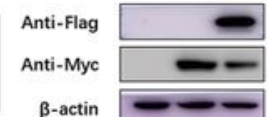

Input $\mid \begin{aligned} & \text { Anti-Flag } \\ & \text { Anti-Myc }\end{aligned}$
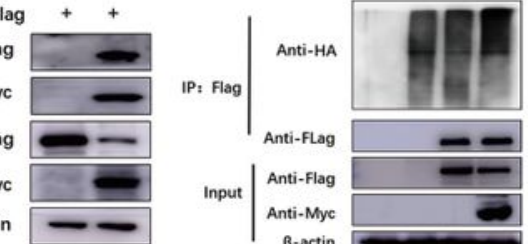

Input Anti-Flag Anti-Myc
$\beta$-actin

1

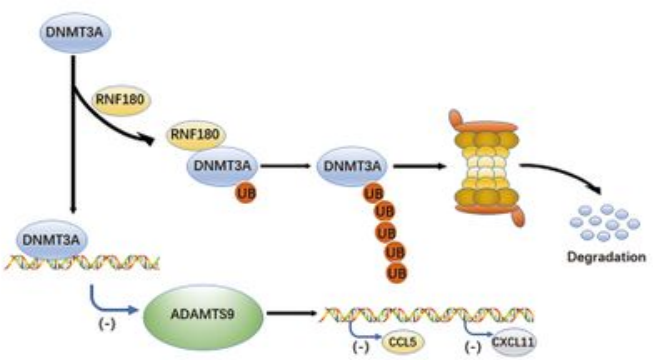

Figure 5 . RNF180 restored ADAMTS9 expression by promoting DNMT3A ubiquitination and degradation.

\section{Figure 5}


RNF180 restored ADAMTS9 expression by promoting DNMT3A ubiquitination and degradation. (A)The expression of RNF180 has a significant positive correlation with the expression of ADAMTS9, according to IHC of TMAs; (B)and the online DEPIA database.(C) The expression of ADAMTS9 was examined by PCR and western blotting method, after transfection with RNF180-overexpression plasmid. (D)The methylation levels of ADAMTS9 DNA promotor after transfection were examined by the MassARRAY analysis. (E) AGS and BGC-823 cells were transfected by RNF180-Flag plasmid or control vector. After $48 \mathrm{~h}$, cells were treated with $100 \mathrm{mg} / \mathrm{ml} \mathrm{CHX}$ at the indicated time point. The DNMT3A, ADAMTS9 and RNF180-Flag proteins were measured through western blotting method. (F) AGS and BGC-823 cells were transfected by RNF180-Flag plasmid or control vector. After $48 \mathrm{~h}$, cells were incubated with $10 \mathrm{uM} \mathrm{MG132}$ for $24 \mathrm{~h}$. The DNMT3A and RNF180-Flag proteins were measured through western blotting method. (G)RNF180-Myc plasmid or control vector was co-transfected transiently with the DNMT3A-Flag plasmid or control vector into HEK293T cells. After transfection 36h, HEK293T cells were treated with 10 uM MG132 for 12h. Then, Co-immunoprecipitation assay was performed to pull down DNMT3A-Flag and RNF180-Myc proteins and the immunoprecipitated proteins were measured through western blotting method. (H) UB-HA and DNMT3A-Flag plasmids were co-transfected with RNF180-Myc plasmid into HEK293T cells. After $36 \mathrm{~h}$, HEK293T cells were treated with 10 uM MG132 for 12h. DNMT3A-Flag protein was immunoprecipitated and measured by western blotting method. The poly-ubiquitination level of DNMT3A was detected by antiHA antibody.

\section{Supplementary Files}

This is a list of supplementary files associated with this preprint. Click to download.

- Supplementarydataset.docx

- SupplementaryTable.docx

- Sfigure2.jpg

- Sfigure5.jpg

- Sfigure6.jpg

- Sfigure3.jpg

- Sfigure1.jpg

- Sfigure4.jpg 